\title{
Hybrid Surface- and Voxel-Based Registration for MR-PET Brain Fusion
}

\author{
Ho Lee ${ }^{1}$ and Helen Hong ${ }^{2, *}$ \\ ${ }^{1}$ School of Electrical Engineering and Computer Science, Seoul National University \\ holee@cglab.snu.ac.kr \\ ${ }^{2}$ School of Electrical Engineering and Computer Science BK21: Information Technology, \\ Seoul National University, San 56-1 Shinlim 9-dong Kwanak-gu, Seoul 151-742, Korea \\ hlhong@cse.snu.ac.kr
}

\begin{abstract}
In this paper, we propose a novel technique of registration using hybrid approach for MR-PET brain image fusion. Hybrid approach uses merits of surface- and voxel-based registration. Thus, our method measures similarities using voxel intensities in MR images corresponding to the feature points of the brain in PET images. Proposed method selects the brain threshold using histogram accumulation ratio in PET images. And then, we automatically segment the brain using the inverse region growing with pre-calculated threshold and extract the feature points of the brain using sharpening filter in PET images. In order to find the optimal location for registration, we evaluate the Hybrid-based Cross-Correlation using the voxel intensities in MR images corresponding to the feature points in PET images. In our experiments, we evaluate our method using software phantom and clinical datasets in the aspect of visual inspection, accuracy, robustness, and computation time. Experimental results show that our method is dramatically faster than the voxel-based registration and more accurate than the surface-based registration. In particular, our method can robustly align two datasets with large geometrical displacement and noise at optimal location.
\end{abstract}

\section{Introduction}

In medical field, image fusion [1] is very useful for early diagnosis as well as for understanding the accurate location of disease by visualizing both anatomical and functional information simultaneously. For example, PET (positron emission tomography) image providing functional information detects changes in the metabolism caused by the growth of abnormal cells before anatomical abnormality, whereas MR (magnetic resonance) image providing anatomical information detects an accurate location of disease due to high resolution. Thus MR-PET image fusion can accurately interpret the location and range of disease with combined information. However, in order to fuse MR-PET brain images, image registration [1-7] determining the relationship of correspondence between two images with different resolution, position, and orientation is necessary.

\footnotetext{
* Corresponding author
} 
The registration methods used to fuse MR-PET brain images can be classified as moment-, surface-, or voxel-based registration. A.P.Dhawan et al. proposed momentbased registration [2]. This method respectively segments the brain from MR and PET images, and computes the center of gravity and its principal axes using zeroth and first order moments with the segmented brain. Registration is then performed by aligning the center of gravity and the principal axes. Since the computation of center of gravity is usually not accurate in blurry ones like PET images, this method is used primarily as a coarse pre-registration. Surface-based registration [3-4] requires delineation of corresponding surfaces in each of the images separately. Y.Hata et al. and L.Y.Hsu et al. proposed the methods to align of the brain surface extracted by automatic segmentation algorithms in MR and PET images. However, these approaches are not easy to accurately segment the only brain in MR images because its intensities are similar to the surrounding non-brain ones. Voxel-based registration [5-7] measures the similarity of all geometrically corresponding voxel pairs within overlapping parts. R.P.Woods et al. proposed a method based on the minimization of the sum of the standard deviation of PET voxel intensities corresponding to narrow ranges of MR voxel intensities [5]. F.Maes et al. proposed mutual information (MI) [7] measuring statistical dependence of intensities in corresponding regions of the two images. However, these methods require enormous processing time due to measuring similarity with all voxel pairs.

In this paper, we propose a novel technique of registration using hybrid approach for aligning MR-PET brain images. Hybrid approach that combines merits of surfaceand voxel-based registration does not attempt to segment the brain from MR images, but only segments the brain in PET images, and then uses the Hybrid-based CrossCorrelation (HCC) to locate the feature points of PET images in the low intensities of voxel between the brain and the surrounding tissue of MR images. Thus, our method aligns MR and PET images by minimizing the similarity of the HCC that uses voxel intensities in MR images corresponding to the feature points of brain in PET images. In our experiments, we evaluate visual inspection, accuracy, robustness, and computation time with software phantom and clinical datasets.

The organization of the paper is as follows. In Section 2, we discuss our registration using hybrid approach in detail: the selection of brain threshold, the automatic segmentation of brain, the extraction of feature points within brain, and the similarity measure and optimization process to find exact geometrical relationship in MR and PET images. In Section 3, experimental results show how the method accurately, robustly, and rapidly aligns in software phantom and clinical datasets. This paper is concluded with a brief discussion of the results in Section 4.

\section{Rigid Registration Using Hybrid Approach}

Fig. 1 shows the pipeline of our method for the registration of MR and PET images. Since MR images have more anatomical information than PET images, MR images are fixed as reference volume and PET images are defined as floating volume. Our method selects the brain threshold using histogram accumulation ratio in PET images. And then, we automatically segment the brain using the $2 \mathrm{D}$ inverse region growing (IRG) with pre-calculated threshold value and extract the feature points of the brain 
using sharpening filter in PET images. The feature points of PET images are transformed to MR images during iterative alignment procedure. At this time, in order to find optimized parameters we evaluate the HCC using the voxel intensities in MR images corresponding to the feature points in PET images. Interpolating PET images at grid positions of MR images is also required for the each iteration depending on the transformation. Since rigid transformation is enough to align the brain base, we use three translations and three rotations about the $x-, y-, z-$ axis. Finally, aligned MR-PET images are displayed by a conventional volume rendering technique.

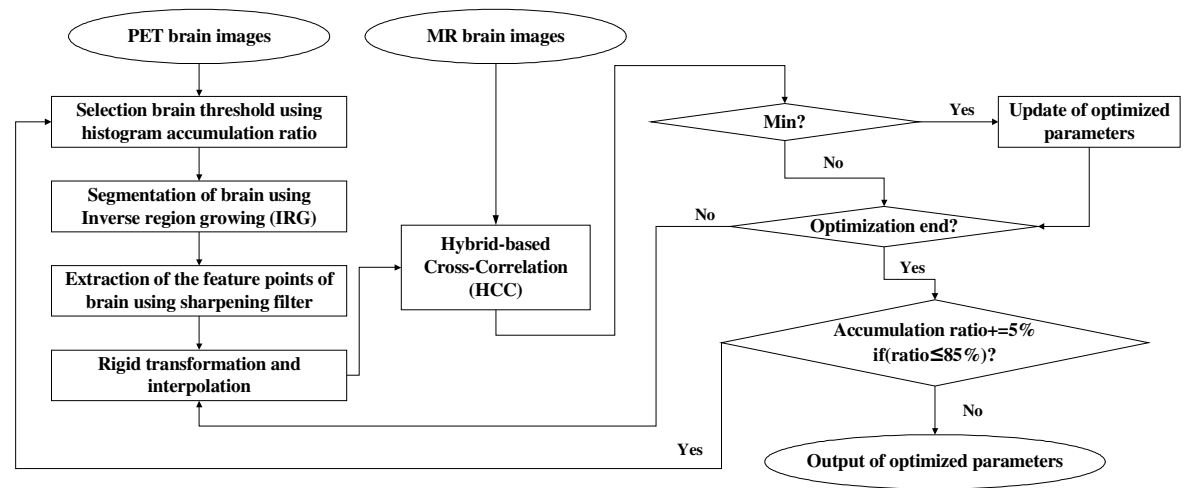

Fig. 1. The pipeline of our method using hybrid approach

\subsection{Selection of the Brain Threshold Using Histogram Accumulation Ratio}

Although the brain area of PET images has higher intensities than non-brain areas such as background and the surrounding tissue, it is difficult to accurately divide into the boundary between the brain and non-brain areas due to blurry properties of PET images. To allow an easy distinction between these areas, we use a histogram accumulation ratio that measures the ratio of non-brain volume of the whole volume.

The computation of ratio accumulates till the highest intensity beginning from the lowest intensity in histogram. If the ratio reaches a certain value, the background area having low intensities is included at first. If it is more accumulative, the surrounding tissue areas are included. At this time, the excluded areas are regarded as brain area, and we select the brain threshold by computing the average of intensity at these areas like following formula (1).

$$
\text { Threshold of brain } \left.=\sum_{i=k}^{n} \frac{H(i) \cdot i}{H(i)} \quad \text { if } \quad \text { Ratio }(\%)=\frac{\sum_{i=0}^{k} H(i)}{\sum_{i=0}^{n} H(i)} \times 100(\%) \geq \operatorname{Init}(\%)\right)
$$

Here, $\mathrm{H}(\mathrm{i})$ is the measure of the number of certain intensity $\mathrm{i}$ in histogram, $\mathrm{k}$ is a intensity when the ratio, or $\operatorname{Ratio}(\%)$, is more than or equal to a certain value as Init $(\%)$, and $n$ is the maximal intensity of the whole intensities. The brain threshold is then selected by averaging from $\mathrm{k}$ to $\mathrm{n}$. 
Fig. 2 is the results applying threshold selected by histogram accumulation ratio when the ratio is increased every 5\% from 50\% to $85 \%$ in PET images. We can select the brain area and a little surrounding tissue when the ratio is $50 \%$. When it is $85 \%$, brain area is eroded.

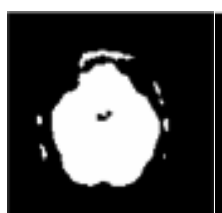

$50 \%$

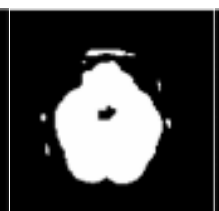

$60 \%$

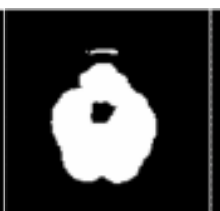

$70 \%$

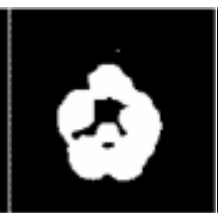

$80 \%$

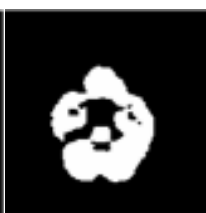

$85 \%$

Fig. 2. The results of brain threshold selected by histogram accumulation ratio

\subsection{Segmentation of the Brain Using 2D IRG and Extraction of Feature Points}

Since the segmentation of brain using threshold-based method [8] can produce holes within the brain, these holes should be filled by morphological operations such as dilation and erosion [8]. However, morphological operations should determine the number of iteration in proportion to the size of holes. In addition computation time is increased by the number of iteration, and the numerous iterations can produce distortion of edge. We propose the 2D IRG for the automatic brain segmentation without these limitations in PET images.

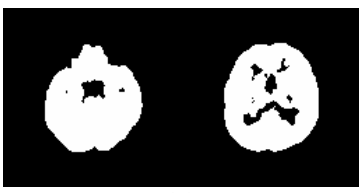

(a)

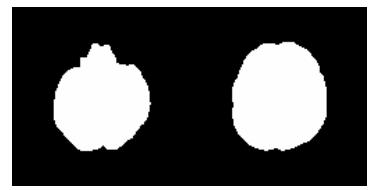

(b)

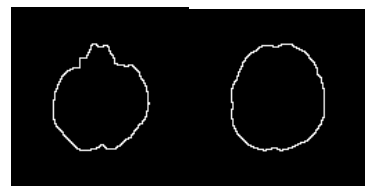

(c)

Fig. 3. Results of the segmentation of brain and the extraction of feature points in each PET slice (a) Threshold-based method (b) Proposed 2D IRG method (c) Feature points of the brain

The $2 \mathrm{D}$ IRG starts by choosing a seed pixel at $(0,0)$ on image and compares it with neighboring pixels. Region is grown from the seed pixel by adding neighboring pixels that are less than threshold selected by histogram accumulation ratio. When the growth of region stops, this region is non-brain areas. Then we simply segment the brain area by inverse operation. Thus the 2D IRG can automatically segment the brain area without inner holes and the distortion of edge by morphological operations in PET images. Fig. 3 shows the results of threshold-based, proposed 2D IRG method, and the feature points of the brain in the each PET slice. In Fig. 3(a), we can easily see inner holes within the brain, whereas proposed 2D IRG method can clearly segment the brain area without inner holes like Fig. 3(b). Fig. 3(c) shows the feature points of the brain extracted by applying a conventional sharpening filter [8]. 


\subsection{Similarity Measure and Optimization}

The similarity measure is used to determine the degree of resemblance of windows in successive frames. Most of voxel-based similarity measures take very long processing time because of considering the voxel intensities of whole volume. We propose the Hybrid-based Cross-Correlation (HCC) that uses the only voxel intensities in MR images corresponding to the feature points in PET images. Our approach reduces computation time because of using the only voxel intensities corresponding to the feature points instead of the voxel intensities of whole volume. The HCC can be defined as formula (2).

$$
H C C=\frac{1}{N_{P E T}} \sum_{i=0}^{N_{P E T}} V_{M R}\left(\operatorname{Tr}\left(P_{P E T}(i)\right)\right)
$$

Here, $N_{P E T}$ is total number of feature points in PET images. $P_{P E T}(i)$ is the position of $i$-th feature point in PET images. $T r$ is rigid transformation matrix transforming feature points of PET images into the coordinate system of MR images. $V_{M R}$ is voxel intensities in MR images corresponding feature points in PET images. In order to search for the optimal location, we find optimized six parameters such as $T_{x}$, $T_{y}{ }^{\prime}, T_{z}{ }^{\prime}, R_{x}{ }^{\prime}, R_{y}{ }^{\prime}, R_{z}{ }^{\prime}$ when the HCC reaches minimum as following formula (3).

$$
\operatorname{Tr}\left(T_{x}{ }^{\prime}, T_{y}{ }^{\prime}, T_{z}{ }^{\prime}, R_{x}{ }^{\prime}, R_{y}{ }^{\prime}, R_{z}{ }^{\prime}\right) \leftarrow \min (H C C)
$$

The both images are initially positioned such that their center coincides and that the corresponding scan axes of both images are aligned and have the same orientation. Powell's multidimensional direction method [7] is then used to minimize the HCC. This method searches for optimal location in the order following $T_{x}, T_{y}, R_{z}, R_{x}, R_{y}, T_{z}$ until the HCC doesn't change any more and iterates over constant number. In this paper, the number of iteration is fixed to five because of converging without update of optimized parameters.

\section{Experimental Results}

All our implementation and test were performed on an Intel Pentium IV PC containing 3.0 GHz CPU and 1.0 GBytes of main memory. Our method has been successfully applied to two clinical datasets and two software phantom datasets as described in Table 1. PET software phantom is generated by dividing into three area such as background, tissue, and brain. The Gaussian smoothing is also added for blurry properties. CT software phantom is generated by dividing into four areas such as background, tissue, brain, and skull. The Gaussian noise is also added to CT of software phantom2 for robustness test. The performance of our method was evaluated with the aspects of visual inspection, accuracy, robustness, and computation time.

For visual inspection in clinical datasets, we provided different display methods such as frame-based, checkerboard-based, edge-based, and three-dimensional data mixing and rendering. As the top row of Fig. 4 is to apply scale parameters only before registration in arbitrary slice, we can see that the brain area of PET images 
misaligns on that of MR images. Whereas we can confirm that the brain area is correctly aligned in the bottom row of Fig. 4 applying optimal parameters. In Fig. $4(d)$, we can see the inner brain area by cutting on arbitrary $3 \mathrm{D}$.

Table 1. Experimental datasets

\begin{tabular}{ccccc}
\hline Dataset & MR/PET & Volume size & Voxel size & Intensity range \\
\hline \multirow{2}{*}{ Patient1 } & MR-T1 & $256 \times 256 \times 94$ & $0.90 \times 0.90 \times 1.50$ & $-1024 \sim 3091$ \\
& FDG-PET & $128 \times 128 \times 40$ & $1.95 \times 1.95 \times 3.33$ & $0 \sim 255$ \\
Patient2 & MR-T2 & $256 \times 256 \times 76$ & $0.78 \times 0.78 \times 1.62$ & $-1024 \sim 3091$ \\
& FDG-PET & $128 \times 128 \times 90$ & $2.00 \times 2.00 \times 2.00$ & $0 \sim 4095$ \\
\multirow{2}{*}{ SW-Phantom1 } & MR & $256 \times 256 \times 94$ & $0.30 \times 0.30 \times 0.3$ & $-1024 \sim 3091$ \\
& PET & $128 \times 128 \times 40$ & $0.32 \times 0.32 \times 0.3$ & $0 \sim 4095$ \\
SW-Phantom2 & MR & $256 \times 256 \times 96$ & $1.00 \times 1.00 \times 1.00$ & $-1024 \sim 3091$ \\
& PET & $128 \times 128 \times 48$ & $2.00 \times 2.00 \times 2.00$ & $0 \sim 4095$ \\
\hline
\end{tabular}

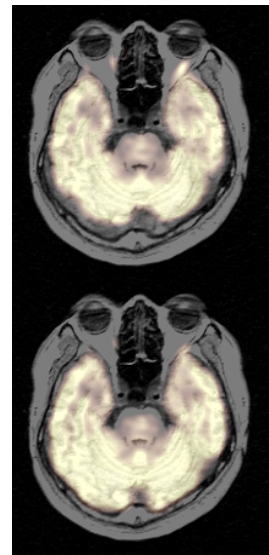

(a)

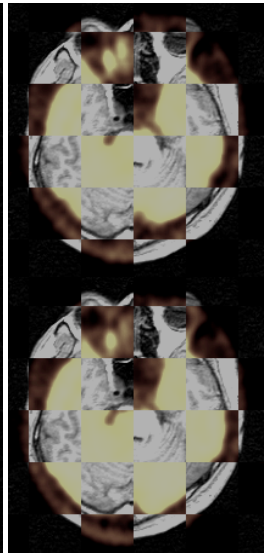

(b)

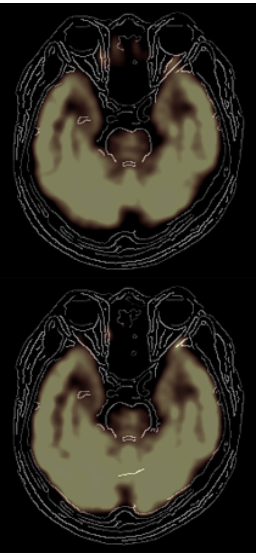

(c)

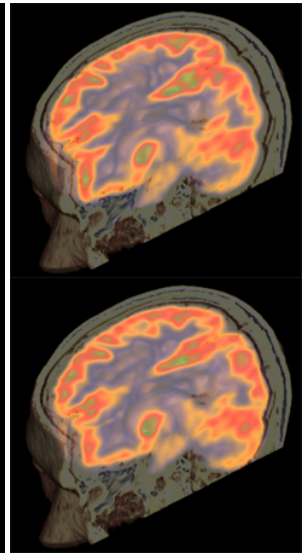

(d)

Fig. 4. The results of visual inspection before and after registration (a) frame-based display (b) checkerboard-based display (c) edge-based display (d) 3D fusion

For accuracy test, we evaluated the root-mean-square-error (RMSE) [8] as formula (4) using the arbitrary transformed software phantom datasets. Then, we compared the RMSE of our method with the one of surface- and voxel-based registration as Table 2. Surface-based registration achieved alignment by measuring correlation between the feature points of the brain extracted by semi-automatic segmentation tools. Voxelbased registration used mutual information method. Our method could keep the quality of the result of voxel-based registration and give more accuracy than the surface-based registration. In Table 2, translation unit is voxel, and rotation one is degree.

$$
R M S E=\sqrt{\frac{1}{6}\left\{\left(T_{x}-T_{x}{ }^{\prime}\right)^{2}+\left(T_{y}-T_{y}{ }^{\prime}\right)^{2}+\left(T_{z}-T_{z}{ }^{\prime}\right)^{2}+\left(R_{x}-R_{x}{ }^{\prime}\right)^{2}+\left(R_{y}-R_{y}{ }^{\prime}\right)^{2}+\left(R_{z}-R_{z}{ }^{\prime}\right)^{2}\right\}}
$$


Table 2. Comparison of RMSE between proposed method and conventional methods

\begin{tabular}{|c|c|c|c|c|c|}
\hline \multirow{2}{*}{\multicolumn{2}{|c|}{$\begin{array}{l}\text { Arbitrary } \\
\text { transformed } \\
\text { parameters }\end{array}$}} & \multicolumn{4}{|c|}{ Optimized parameters } \\
\hline & & & Proposed & \multirow{2}{*}{$\begin{array}{c}\text { Surface- } \\
\text { based }\end{array}$} & \multirow{2}{*}{$\begin{array}{c}\begin{array}{c}\text { Voxel- } \\
\text { based }\end{array} \\
-2.438\end{array}$} \\
\hline$T_{x}$ & -2.4 & $T_{x}$ & -2.375 & & \\
\hline$T_{y}$ & -3.6 & $T_{y}^{\prime}$ & -3.563 & -1.813 & -3.500 \\
\hline$T_{z}$ & -0.5 & $T_{z}$ & -0.563 & 0.000 & -0.438 \\
\hline$R_{x}$ & -2.1 & $R_{x}$ & -2.188 & 0.000 & -2.100 \\
\hline$R_{y}$ & 1.0 & $R_{y}$ & 1.000 & 0.000 & 1.000 \\
\hline$R_{z}$ & -4.0 & $R_{z}$ & -4.000 & -1.688 & -4.000 \\
\hline \multicolumn{2}{|c|}{ RMSE } & & 0.047796 & 1.69186 & 0.050478 \\
\hline
\end{tabular}

For robustness test, we evaluated whether the HCC searches for optimal location in software phantom datasets with a large geometrical displacement and noise. As shown in Fig. 5, we can confirm that our method searches for optimal location where the HCC is a minimum at 0 voxel or degree. In particular, our result robustly converged on optimal location even in software phantom dataset 2 added Gaussian noise.

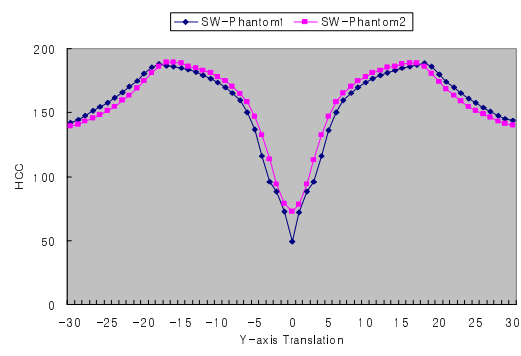

(a)

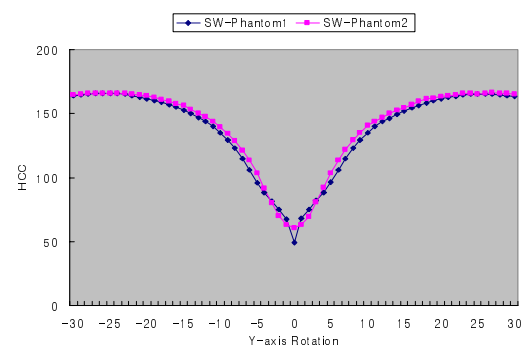

(b)

Fig. 5. Robustness test (a) Translation in the y-direction (b) Rotation in the y-direction

The total computation time is summarized by comparing our method with the conventional surface- and voxel-based registration in Table 3. Our method was dramatically faster than the voxel-based registration and was suitable for clinical use.

Table 3. Comparison of computation time between our method and conventional methods

\begin{tabular}{cccc}
\hline & & & $(\mathrm{sec})$ \\
\hline Patient1 & 23.8 & 3.3 & 648.1 \\
Patient2 & 43.1 & 7.2 & 1755.7 \\
SW-phantom1 & 24.2 & 3.4 & 639.8 \\
SW-phantom2 & 25.4 & 4.1 & 721.5 \\
\hline
\end{tabular}




\section{Conclusion}

We have developed a novel technique of registration using hybrid approach that combines merits of surface- and voxel-based registration for MR-PET brain fusion. Our histogram accumulation ratio was suitable for the selection of threshold in blurry ones such as PET images. The 2D IRG automatically segmented the brain without inner holes in PET images. In particular, the HCC could reduce computation time because of considering the only voxel intensities of MR images corresponding to the feature points extracted from PET images. Experimental results showed that our method was dramatically faster than the voxel-based registration and more accurate than the surface-based registration. Moreover, our method could robustly align two datasets with large geometrical displacement and noise at optimal location.

\section{Acknowledgements}

This work was supported in part by a grant B020211 from Strategic National R\&D Program of Ministry of Science and Technology and a grant 10014138 from the Advanced Technology Center Program. The ICT at Seoul National University provides research facilities for this study.

\section{References}

1. J.B.A.Maintz, M.A.Viergever, A survey of medical image registration, Medical Image Analysis, Vol.2, Iss.1 (1998) 1-36.

2. A.P.Dhawan, L.K.Arata, Alejandro V.Levy, and Joseph Mantil, Iterative Principal Axes Registration Method for Analysis of MR-PET Brain Images, IEEE Transactions on Biomedical Engineering, Vol.42, No.11 (1995) 1079-1087.

3. Y.Hata, S.Kobashi, S.Hirano, and M.Ishikawa, Registration of Multi-Modality Medical Images by Soft Computing Approach, ICONIP 6th International Conference on Neural Information Processing, Vol.3 (1999) 878-883.

4. L.Y.Hsu, M.H.Loew, Fully automatic 3D feature-based registration of multi-modality medical images, Image and Vision Computing Vol.19 (2001) 75-85.

5. R,P,Woods, J.C.Mazziotta, S.R.Cherry, MRI-PET registration with automated algorithm, Journal of Computer Assisted Tomography, Vol.17 (1993) 536-546.

6. J.Cizek, K.Herholz, S.Vollmar, R.Schrader, J.Klein, W-D, Heiss, "Fast and robust registration of PET and MR images of human brain," Neuroimage, Vol.22, Iss.1 (2004) 434-442.

7. F.Maes, A.Collignon, G.Marchal, P.Suetens, Multimodality Image Registration by maximization of Mutual Information, IEEE Transaction on Medical Imaging, Vol.16, No.2 (1997) 187-198.

8. R.G.Gonzalez, R.E.Woods, Digital Image Processing, 1st Ed. (1993). 